\title{
Thermal response to heat fluxes of the W7-AS divertor surface submitted to surface modification under high temperature treatment
}

\author{
D. Hildebrandt, A. Dübner, H. Greuner*, A. Wiltner* \\ Euratom Association Max-Planck-Institut für Plasmaphysik, \\ Teilinstitut Greifswald Wendelsteinstraße 1, 17491 Greifswald, Germany \\ * Teilinstitut Garching Boltzmannstr.2, 85748 Garching, Germany
}

\begin{abstract}
Some target tiles of the W7-AS divertor has been investigated with respect to their thermal behaviour at the surface during power loading with well defined heat fluxes in the Gladis facility. The primary aim was to examine uncertainties in the determination of heat fluxes derived from IR-thermography during operation of W7-AS. It is found that the derived heat flux profiles are strongly influenced by the local distribution of plasma-deposited contamination analyzed by AES and SIMS. With the observed actual surface conditions characterized by redeposited contamination equivalent up to about $1 \mu \mathrm{m}$ thickness, the heat fluxes were partially overestimated up to a factor of 4 during operation of W7-AS. This uncertainty is observed to be significantly reduced after heat treatment at temperatures beyond $700{ }^{\circ} \mathrm{C}$ attained at power flux densities of $10.5 \mathrm{MW} / \mathrm{m}^{2}$ and durations longer than 5 seconds.
\end{abstract}

JNM Keywords: Carbon, Divertor Materials, Heat Treatment, Thermodynamic Properties PSI18 Keywords: W7-AS, Divertor tiles, Thermal conductivity, IR-thermography, Power flux PACS codes: $44.10,44.40,52.40,52.55$

Corresponding author: D. Hildebrandt, Max-Planck-Institut für Plasmaphysik, Teilinstitut Greifswald, Wendelsteinstr.1, 17491 Greifswald, Germany e-mail address: dieter.hildebrandt@ipp.mpg.de 


\section{Introduction}

In 2000 the stellarator W7-AS was equipped with a new open divertor. During operation until 2002, thermographic and calorimetric measurements were routinely carried out to diagnose the power loading of the target elements [1,2]. The energy deposited during the discharges onto the target tiles derived from the thermographic data is found to be systematically higher up to a factor of two compared with values measured via calorimetry [1,3].

It is considered that the evaluation of power flux densities derived from the evolution of the surface temperature distribution suffered from uncertainties of the actual thermal properties at the surface of the target tiles. These properties are known to be modified continuously by erosion and deposition processes during plasma exposure.

After shut down of W7-AS the divertor tiles were removed. The emissivity and the thermal resistance of the plasma-exposed surface have been examined in order to estimate the error made by earlier evaluations of thermographic data during operation using virgin material parameters.

Well-defined heat fluxes have been applied to the tile surfaces and the response of the surface temperature is observed. The actual power flux densities are compared with corresponding values derived from the temporal evolution of the measured surface temperature distribution as done during machine operation.

This paper presents results of experiments involving well-defined heat pulses by ion beam radiation on selected target tiles of the lower divertor. In particular, the surface modification due to thermal conditioning observed earlier on divertor tiles of ASDEX-Upgrade [4] is also considered.

Therefore the surface contamination on the tiles has also been investigated by Secondary Ion Mass Spectrometry (SIMS) and Auger Electron Spectroscopy (AES).

\section{Experimental}

The divertor tiles are prepared from the CFC material SEBCARB N11. Their surface morphology is characterized by a mean surface roughness of $15 \mu \mathrm{m}$ [4]. The thermal conductivity of the CFC material at room temperature has been determined for all three directions in separate heat pulse experiments [3]. The main interaction zones of the plasma on the horizontal targets observed by thermography, $\mathrm{H}_{\alpha}$-diagnostics and Langmuir probes are two coloured helical stripes. These stripes indicate redeposited material and are characterized by an emissivity of 1 in the far infrared region while the divertor material outside the interaction zone has an emissivity of about 0.85 (see fig.1). 
The experiments have been performed on the test facility GLADIS [5] with a hydrogen beam of $30 \mathrm{keV}$ focused to a spot with Gaussian shaped profile and a standard deviation of about $70 \mathrm{~mm}$. Heat fluxes with central densities up to $10.5 \mathrm{MW} / \mathrm{m}^{2}$ and pulse durations up to $5 \mathrm{sec}$, respectively have been applied. Mass spectrometry measurements allowed to observe gas desorption from the tiles during the heat pulses. The experiments were conducted on the tiles $4,5,12$ and 13.

Surface temperature evolutions during and after the heat pulses were measured by microbolometer cameras with $320 \times 240$ and $640 \times 480$ pixels operating in the wavelength region of 8-15 $\mu \mathrm{m}$ with frame rates of $50 \mathrm{~Hz}$.

In order to investigate thermal conditioning effects [4] we started the experiments with a low power density pulse of $4 \mathrm{MW} / \mathrm{m}^{2}$ and $1 \mathrm{sec}$ duration. For two successive pulses the power density and pulse duration was increased to $10.5 \mathrm{MW} / \mathrm{m}^{2}$ and $5 \mathrm{sec}$, respectively. In this case the predicted surface temperature exceeds $1200 \mathrm{~K}$ and we finished with the low power density pulse from the beginning.

Surface analysis was done on divertor tile 5 before and after the ion beam experiment. For SIMS-depth profiling an Ar-beam with an energy of $10 \mathrm{keV}$ was used. The diameter of the analyzing beam was smaller than $5 \mu \mathrm{m}$. This allows to take 2D-images of the surface contamination. AES was done with an electron beam of $3 \mathrm{keV}$ and a diameter of about $1 \mu \mathrm{m}$.

\section{Modelling}

In order to calculate the power flux densities from the measured temporal evolution of the surface temperature distribution, the finite element code ANSYS (www.ansys.com) was used in 2D geometry. The toroidal dependence is neglected. For the calculations, the following temperature dependent parameters for the CFC material given by the manufacturer have been used for the thermal conductivity $\lambda$ and the specific heat capacity c:

$$
\lambda_{\mathrm{x}, \mathrm{z}}(T)=310 \cdot \mathrm{e}^{\left(-\frac{T}{315}\right)}+105 \quad \text { and } \quad \mathrm{c}(T)=2060 \cdot\left(1-\mathrm{e}^{(-0.00166 \cdot T)}\right)
$$

with $\lambda$ in $\mathrm{W} /(\mathrm{K} \cdot \mathrm{m})$ and $\mathrm{c}$ in $\mathrm{J} /(\mathrm{kg} \cdot \mathrm{K})$.

The tile was discretized with the same spatial resolution as the infrared camera delivered, which resulted in an element size of $0.9 \mathrm{~mm}$. The shape function of the elements was of quadratic order. The temperature boundary condition was of Dirichlet-kind, and directly applied at the top layer of the tile. The midside nodes yielded to a better approximation of the temperature field inside the tile, which was characterized by significant temperature gradients 
in the vertical direction. The temperature was applied at the same locations as measured by the infrared camera. No interpolation of the boundary conditions for the midside nodes was done. The midside nodes fulfilled the Neumann-condition with a zero flux out of this surface. It was assumed that the equilibrium is established quickly between two edge nodes of an element, due to the high spatial resolution of less than a millimeter. This limits the horizontal flux to the midside nodes of the surface to small values, which cannot reduce the flux into the tile significantly.

For the output of the heat flux at the surface the same nodes were used as for the temperature application. The flux perpendicular $\left(q_{z}\right)$ and parallel $\left(q_{x}\right)$ to the surface were written out, as well as the resulting thermal flux $q=\sqrt{q_{x}^{2}+q_{z}^{2}}$.

\section{Results}

\subsection{Contamination}

Fig. 2 shows the surface temperature pattern on 3 different tiles as the response of a nearly uniform heat flux about $30 \mathrm{~ms}$ after the beginning of the first and the last pulse with 4 $\mathrm{MW} / \mathrm{m}^{2}$. Between the first and the last pulses, two further pulses with $10.5 \mathrm{MW} / \mathrm{m}^{2}$ with durations up to $5 \mathrm{sec}$ were performed.

The surface temperature distribution was found to be quite non-uniform during the first pulse. (see fig.2). Each divertor tile exhibits its characteristic surface temperature pattern depending on the local surface conditions. The two stripe zones with a width of a few mm already seen in fig. 1 show a much higher temperature excursion than the surrounding indicating a surface layer of redeposited material with high thermal resistance. In fact, surface analysis by SIMS and AES showed a mixed material on all surface parts of the tiles consisting of carbon, boron, iron, deuterium and oxygen with the largest amount within the stripes prior to the heat pulses. SIMS depth profiles of the contaminants are found with a long tail due to the high surface roughness of the substrate. The contamination is not a homogeneous layer but particularly located in surface depressions [6]. AES yielded surface concentrations of $66 \% \mathrm{C}, 20 \% \mathrm{O}, 9 \%$ Fe and 5\% B ( $\mathrm{H}$ and $\mathrm{D}$ can not be detected by AES). The amount of this contamination corresponds approximately to a layer varying in thickness between $200 \mathrm{~nm}$ outside the stripes and about 1-2 $\mu \mathrm{m}$ within the stripes. Taking a heat conduction coefficient of this contamination obtained for the deposit on ASDEX-Upgrade CFC-divertor tiles of $0.1 \mathrm{~W} / \mathrm{Km}$ [7] an excellent agreement between calculated and measured temperature values is also found in laser experiments performed on the deposit zones of the W7-AS tiles (unpublished). This 
conductivity is coherent as well with the temperature of fig. 3 during the first pulse (4 $\left.\mathrm{MW} / \mathrm{m}^{2}\right)$.

As can be seen in fig. 2 the temperature pattern during the last pulse with $4 \mathrm{MW} / \mathrm{m}^{2}$ is much more uniform. In particular the two stripe zones can hardly be recognized. Obviously, the thermal resistance at the surface have been significantly reduced in the stripe zones. In fact, a continuous harmonization of the temperature pattern was clearly observed during the first $10.5 \mathrm{MW} / \mathrm{m}^{2}$ heat pulse when the surface temperature achieved values beyond $700{ }^{\circ} \mathrm{C}$ within the stripe zones. This is demonstrated in fig. 3. It shows the surface temperature evolution inand outside the stripe zones of tile 13 for the two first pulses. The reduction of the thermal resistance was found to be correlated with gas desorption of mass $4\left(\mathrm{D}_{2}\right), 20\left(\mathrm{CD}_{4}\right)$ and 18 $\left(\mathrm{H}_{2} \mathrm{O}\right)$. Fig. 4 reveals that gas desorption occurred during the first two pulses. A minor amount of deuterium is already desorbed during the first pulse with a power density of 4 $\mathrm{MW} / \mathrm{m}^{2}$ where a surface temperature up to $300{ }^{\circ} \mathrm{C}$ was observed within the stripe zones. The dominant amount of deuterium was desorbed during the second pulse with $10.5 \mathrm{MW} / \mathrm{m}^{2}$. The desorption peak occurred at about $1.5 \mathrm{sec}$. At that time the attained surface temperature was about $700{ }^{\circ} \mathrm{C}$ (compare fig. 3 and 4). No further significant gas release was observed for the successive pulses 3 and 4. SIMS and AES analysis performed after the heat pulses showed that deuterium and oxygen were widely desorbed while the other impurities as boron and iron are still present at the surface. Within the stripe zone the surface composition has been found by AES to be $81 \% \mathrm{C}, 8 \% \mathrm{Fe}, 8 \% \mathrm{~B}$ and $3 \% \mathrm{O}$.

\subsection{Heat flux calculation}

Fig. 5 presents the spatiotemporal heat flux densities calculated from the temporal evolution of the measured temperature distribution for the first pulse with $4 \mathrm{MW} / \mathrm{m}^{2}$ on tile 5 . Within the stripe zones the calculated heat flux is strongly enhanced compared to that of the surrounding parts and it decreases with time from $14 \mathrm{MW} / \mathrm{m}^{2}$ at the beginning to $7 \mathrm{MW} / \mathrm{m}^{2}$ at termination of the heat pulse. The actual heat flux density profile about $30 \mathrm{~ms}$ after beginning is compared with the calculated values for the first and last heat pulse in fig. 6. It demonstrates that the calculated heat flux density within the stripes is overestimated by a factor up to 4 for the first pulse (fig.6a) while it agrees satisfactorily with the actual heat flux density for the surrounding. This holds also for the total area with the exception of a small surface part at $\mathrm{x}=$ $0.13 \mathrm{~m}$ for the last pulse (fig. $6 \mathrm{~b}$ ) where the computed heat flux density is still enhanced by a factor of 2 after the heat treatment during pulse 3 and 4. Obviously, the thermal conductivity is still somewhat influenced by remained contamination also indicated by the IR-emissivity (see fig 2). The highest local temperature values observed during pulse 3 and 4 are given in fig. 6 a for different regions with significant contamination. 


\section{Conclusions}

The surface of the divertor tiles was continuously modified by erosion and deposition processes during boronization procedures and plasma operation in W7-AS. The involved variation in the thermal resistance of the surface introduces uncertainties in the determination of power flux densities using surface temperature values. The present investigation on the removed target tiles with well-defined heat fluxes using ion beams clearly demonstrate that the found contamination of about $1 \mu \mathrm{m}$ thickness has to be taken into account. The derived heat flux profiles are strongly influenced by the local distribution of this contamination.

The power fluxes previously computed for the stripe zones of local deposition by numerical code calculations during operation of W7-AS are partially overestimated by a factor up to 4 at the beginning of the heat pulse and up to 2.5 at termination. This fact can account for the discrepancies in the comparison of thermographic and calorimetric data observed during operation of W7-AS.

The deposition zones are identified as the main interaction region of the plasma with the divertor surface. The thermal resistance of the contamination is significantly reduced by gas desorption when the surface was heated up to temperatures beyond $700{ }^{\circ} \mathrm{C}$ during pulses with $10.5 \mathrm{MW} / \mathrm{m}^{2}$ and a duration of $5 \mathrm{sec}$. However, such surface temperatures were never observed for $1 \mathrm{sec}$ during plasma operation of W7-AS.

\section{References}

[1] F. Gadelmeier et al., Plasma Phys. Control. Fusion 46 (2004) 711

[2] M. Hirsch et al., Plasma Phys. Control. Fusion 50 (2008) no5

[3] D. Hildebrandt et al., J. Nucl. Mater. 313-316 (2003) 738

[4] D. Hildebrandt et al., J. Nucl. Mater. 337-339 (2005) 1064

[5] H. Greuner et al., Fusion Eng. Des. 75-79 (2005) 345-350.

[6] D. Hildebrandt et al., Physica Scripta Vol.T81 (1999) 25

[7] D. Hildebrandt et al., J. Nucl. Mater. 363-365 (2007) 1221 
Figure captions

Fig.1: Thermal image of divertor tile 5 after uniform warming indicating the apparent temperature and derived emissivity values inside and outside the stripe zones

Fig.2: Surface temperature pattern on 3 different tiles as the response of a nearly uniform heat flux about $30 \mathrm{~ms}$ after the beginning of the first (left side) and the last pulse (right side) with 4 $\mathrm{MW} / \mathrm{m}^{2}$. Between the first and the last pulse 2 further pulses with $10.5 \mathrm{MW} / \mathrm{m}^{2}$ with durations up to $5 \mathrm{sec}$ were performed.

Fig.3: Surface temperature evolution in- and outside the stripe zones of tile 13 for the two first pulses indicating the convergence of the temperatures for values beyond $700{ }^{\circ} \mathrm{C}$.

Fig.4: Desorption of mass 4 (deuterium) occurred during all pulses applied on tile 13. It reveals that the dominant amount of gas is desorbed during the first $10.5 \mathrm{MW} / \mathrm{m}^{2}$ pulse at temperatures of about $700{ }^{\circ} \mathrm{C}$ (compare with fig 3 ). The background signal is $1.4 * 10^{-11} \mathrm{~A}$.

Fig.5: Spatiotemporal heat flux densities calculated from the temporal evolution of the measured temperature distribution for the first pulse with $4 \mathrm{MW} / \mathrm{m}^{2}$ on tile 5

Fig.6: Comparison of the calculated heat flux density profile about $30 \mathrm{~ms}$ after beginning of the heat pulse with the actual values for the first and last pulse on tile 5 


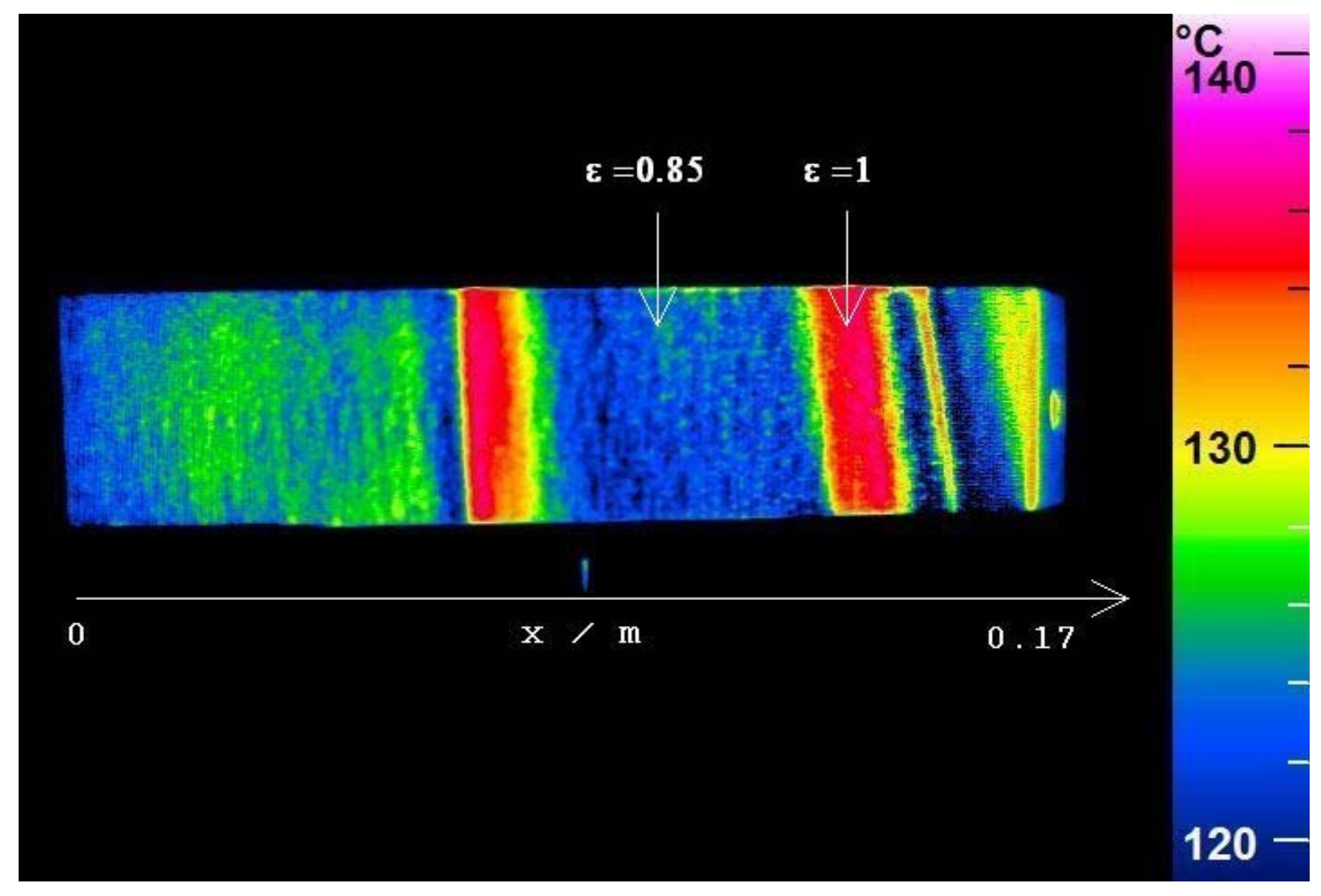

Fig. 1 


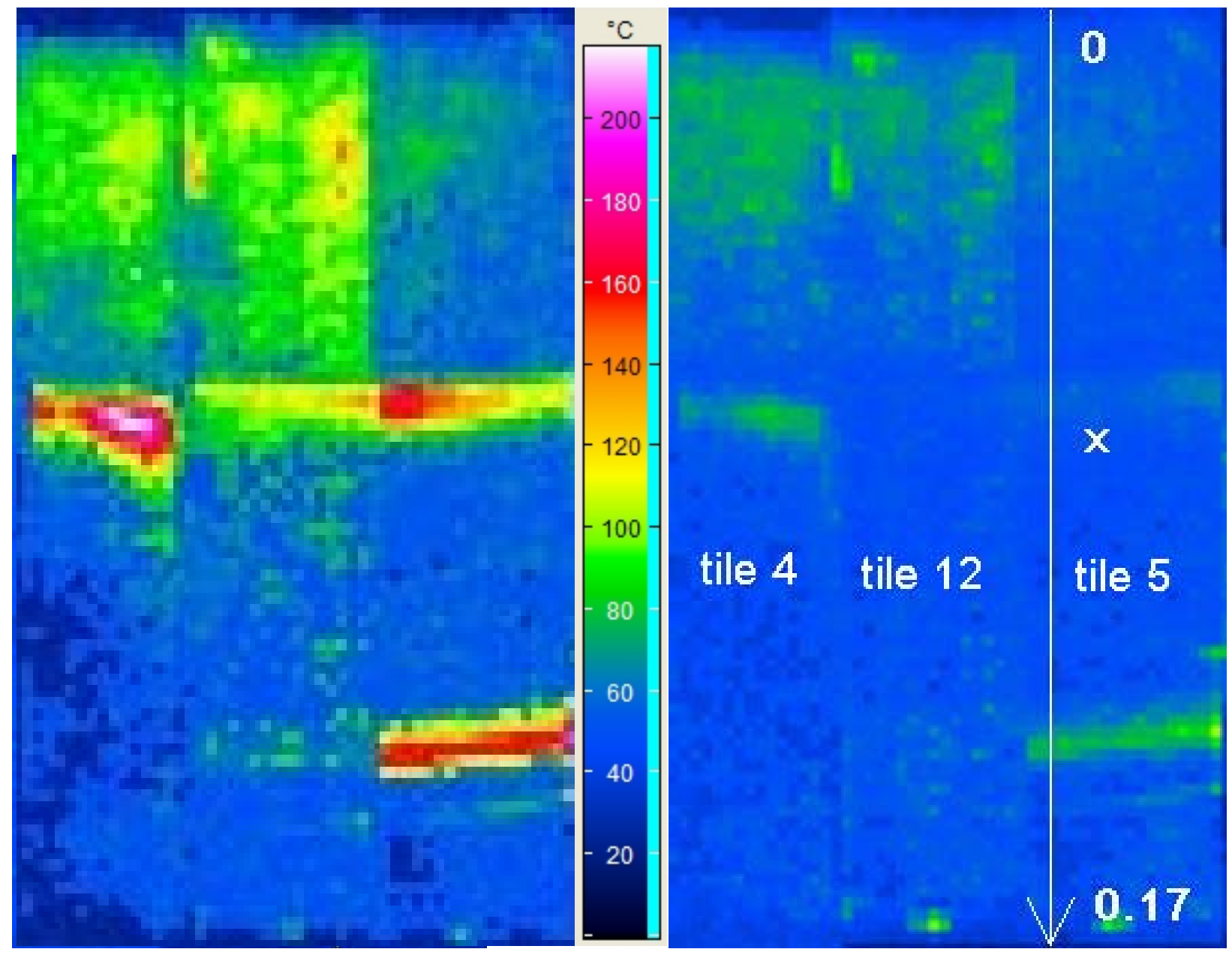

Fig. 2 


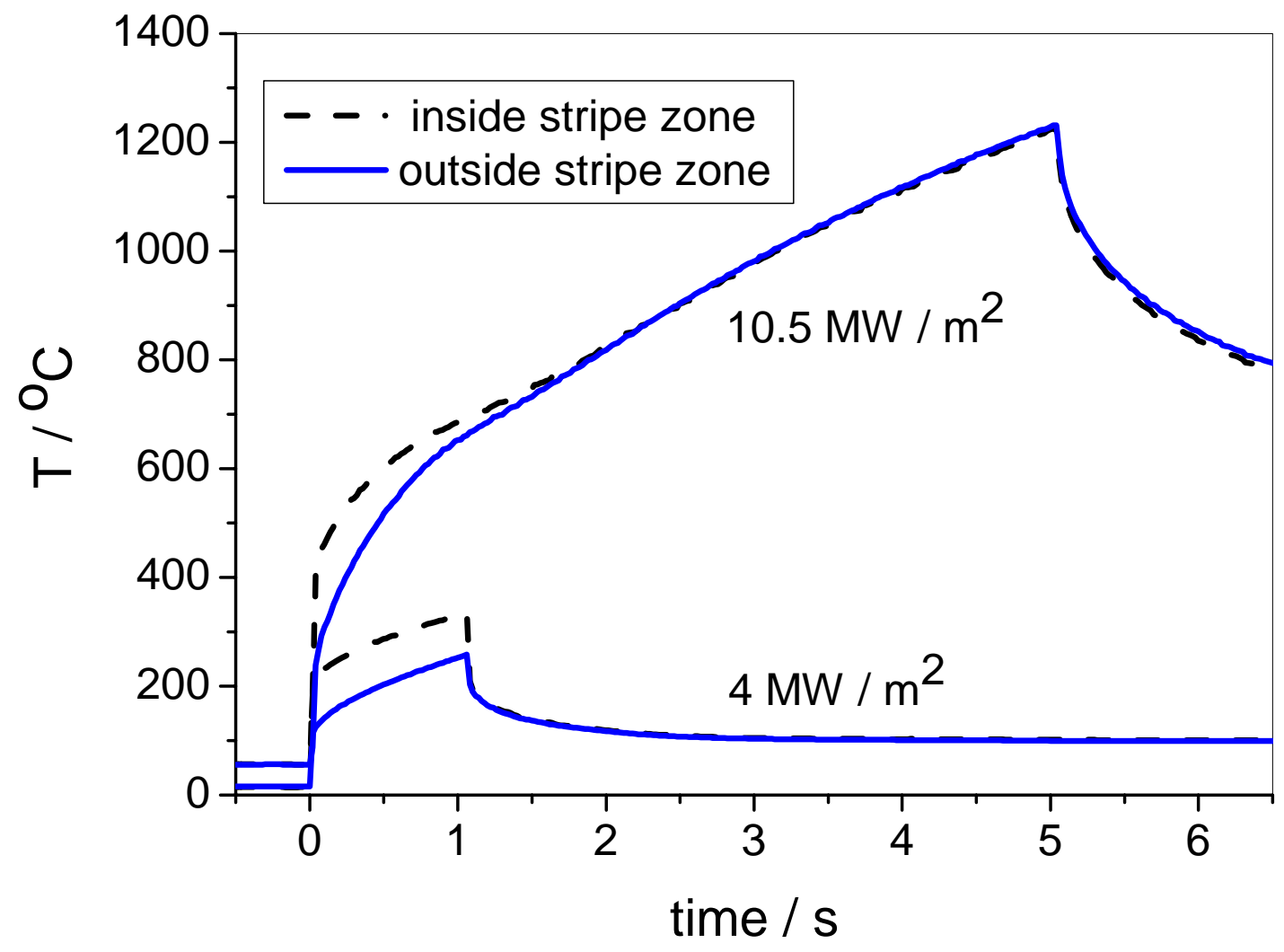

Fig. 3 


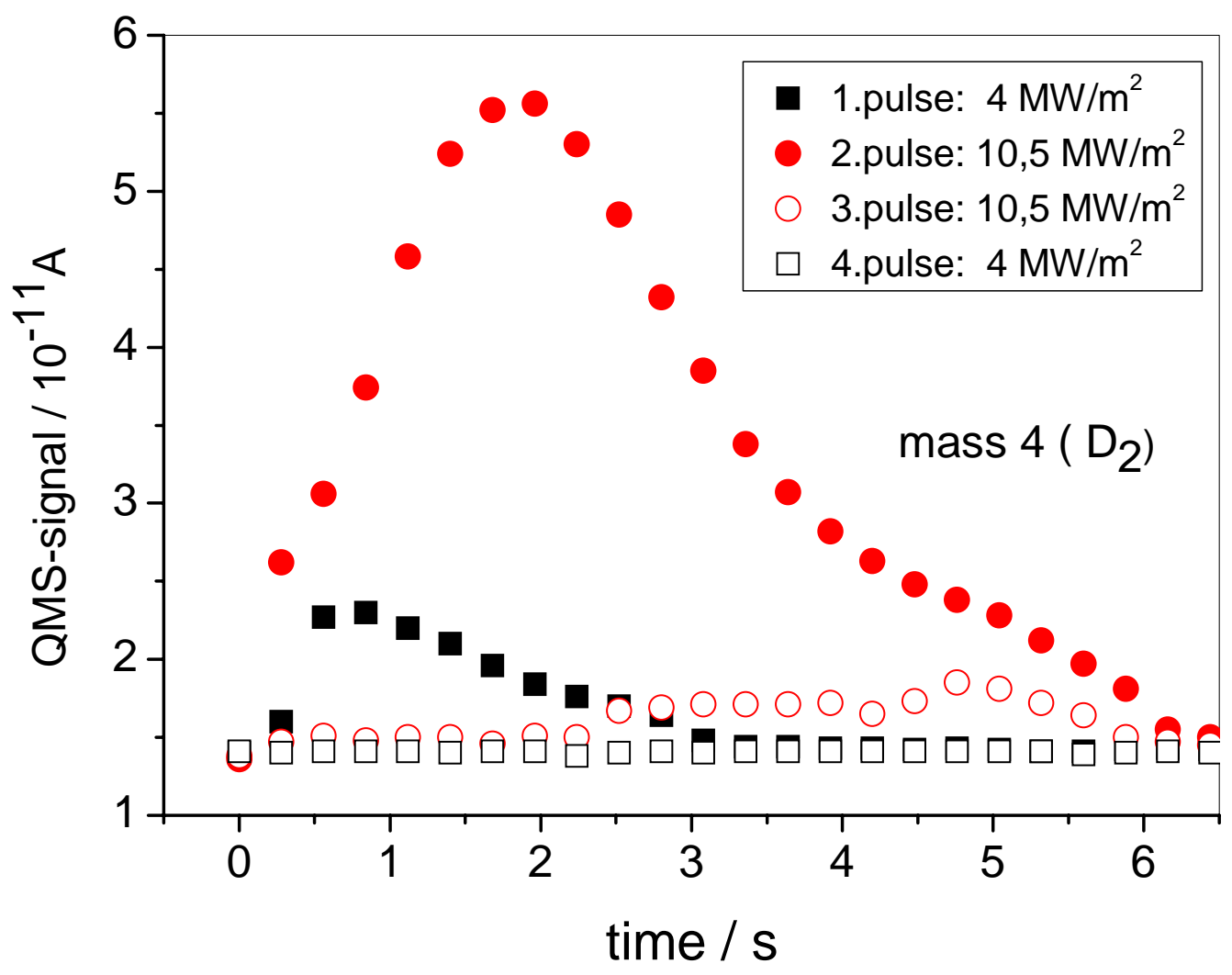

Fig.4 


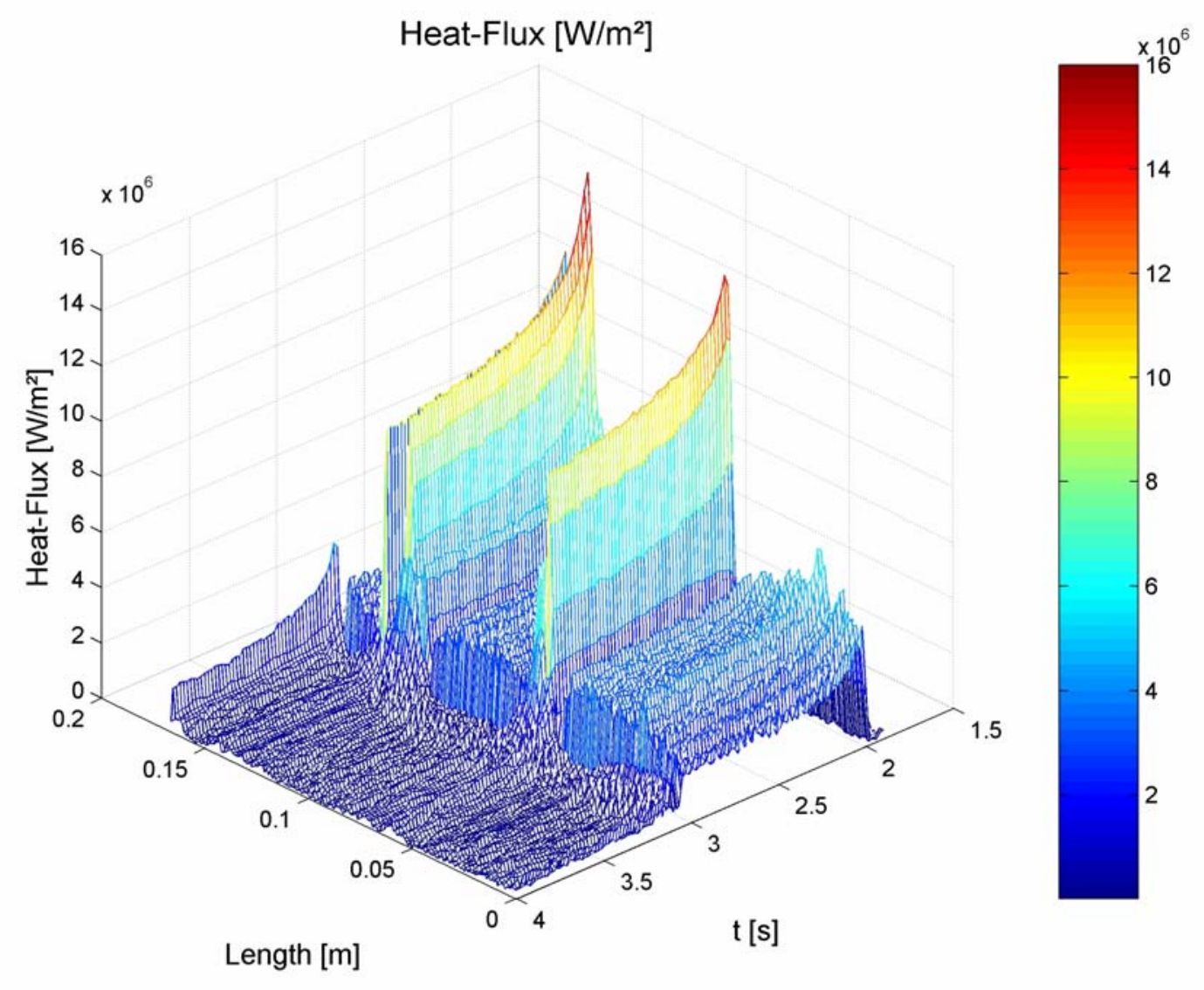

Fig.5 

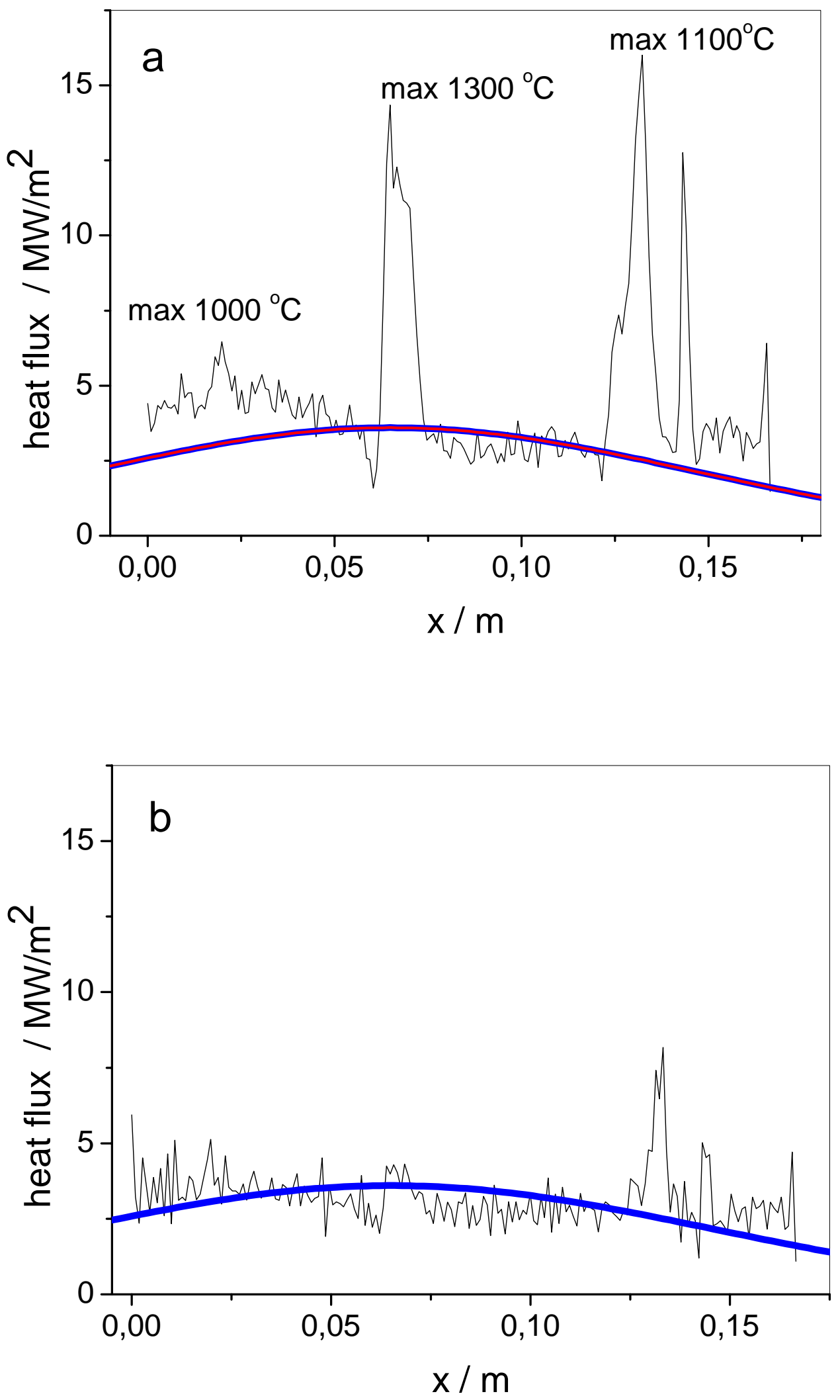

Fig.6 